\title{
CONVERTIR LA EVALUACIÓN EN UNA OPORTUNIDAD PARA APRENDER. UNA PROPUESTA PARA LA ASIGNATURA DE LENGUA ESPAÑOLA
}

\author{
Ana Blanco Canales \\ Universidad de Alcalá
}

\begin{abstract}
RESUMEN: Las nuevas metodologías de enseñanza universitaria exigen cambios urgentes en los sistemas de evaluación, pues es un hecho indiscutible que la evaluación es, finalmente, la que determina lo que aprenden los alumnos y cómo lo aprenden. La evaluación formativa integrada se presenta, en estos momentos, como un planteamiento docente con numerosísimas posibilidades de aplicación. Resulta de especial interés su capacidad para generar contextos de aprendizaje multifuncionales (adquisición de competencias, desarrollo de estrategias de aprendizaje, calificación de resultados, evaluación de los procesos...), en los que la participación y compromiso del alumno surgen de manera natural. Con objeto de valorar su factibilidad, el impacto sobre el aprendizaje y su contribución a la resolución de algunas dificultades hemos desarrollado una propuesta de evaluación global para la asignatura de Lengua Española, que describimos con detalle a lo largo de este trabajo. Presentaremos los fundamentos sobre los que se apoya, los objetivos que persigue, la metodología de trabajo que requiere así como los resultados obtenidos en su implementación. Asimismo, ofrecemos todo el repertorio de actividades que componen la propuesta, pues, con los cambios convenientes, podrá ser de utilidad para otros profesores.
\end{abstract}

ABSTRACT: New methodologies of teaching developed in university classrooms demand urgent change in the systems of evaluation, because it is inexcusable that the evaluation is in the end, what determines what the students learn and how they learn. The formative integrated assessment presents, in moments, as a student platform with numerous possibilities for application. Interestingly, this generates multifuntional contexts for learning (acquisition of competencies, development of learning strategies, assessment of results, evaluation of the processes...), of which the participation and engagement of the student increases in a natural manner. With the objective to evaluate the effectiveness, the impact over the learning and its contribution to resolving some difficulties we have developed a proposal of 
global evaluation for the Spanish language course, which we describe with detail throughout this work. We present the supporting fundamentals, the objectives that follow it, the required methodology as well as the results obtained upon its implementation. Likewise, we offer the complete repertory of activities that make up the proposal, since with changes and simple adaptations, they can be useful to other professors.

PALABRAS CLAVE: Evaluación formativa, innovación docente, estrategias de aprendizaje, lengua española.

KEYWORDS: Formative assessment, teaching innovation, learning strategies, Spanish language.

Recibido: $14 / 09 / 2010$

Aceptado: 16/12/2010

\section{INTRODUCCIÓN: LA EVALUACIÓN FORMATIVA}

El nuevo escenario europeo creado por la convergencia hacia el Espacio Europeo de Educación Superior ha traído consigo una profunda reformulación de las metodologías docentes, centradas ahora más en el aprendizaje que en la enseñanza. Como consecuencia, el alumno pasa a ocupar una posición central en el proceso, convirtiéndose en el protagonista y verdadero impulsor de su aprendizaje; al mismo tiempo, el tradicional enfoque docente, articulado en torno a los contenidos de la materia, es sustituido por otro orientado a las competencias.

Para cumplir con eficacia los objetivos y los retos que estos planteamientos conllevan, se hace imprescindible un replanteamiento de la evaluación, tanto de su naturaleza y función, como de los instrumentos en que se apoya, pues es un hecho indiscutible que es la evaluación la que determina, finalmente, lo que deben aprender los alumnos y la manera que tienen de aprenderlo. De esta forma, cualquier cambio en las estrategias y metodologías docentes debe contemplar -y empezar por- cambios en las formas de evaluación. Son muchos ya los estudios que demuestran que el cómo aprenden los alumno está estrechamente vinculado a la forma del examen, y que por más que en el programa se contemplen, junto con los contenidos, el desarrollo de determinadas habilidades o destrezas, si el examen, finalmente, se centra en los primeros y posee unas características determinadas, el alumno acomodará a ello su aprendizaje. Así, de nada nos sirve trabajar con los estudiantes el pensamiento crítico y reflexivo, la creatividad, la capacidad para relacionar y aplicar conocimientos a diversas situaciones si al final valoraremos sus logros con un examen tipo test, por ejemplo. Aunque los estudiantes estén muy motivados, sean muy participativos, manifiesten un alto grado de compromiso con un aprendizaje real, no cabe duda de que optarán por la forma de aprender que mejor solucione el examen; si este consiste en un test, se decantarán por un aprendizaje memorístico, de escasa pervivencia. Así, los estudiantes adoptarán un enfoque superficial si perciben que eso es lo que la asignatura y la evaluación requieren o que es el enfoque que en mejor situación les pone para responder a las demandas de la asignatura (Kember, 2000). De todo esto se desprende que, si queremos modificar la forma de estudiar y de aprender de nuestros alumnos, la manera más rápida y eficaz de conseguirlo es cambiando la evaluación 
(Elton y Laurillard, 1979). En este sentido, y aunque resulte algo radical, estamos de acuerdo con Brown (2006) cuando afirma que:

Nuestro modo de evaluar a los alumnos en Educación Superior tiene tal impacto en el aprendizaje de los alumnos, que necesitamos repensar todo el proceso de programación y diseño del currículum y traer la evaluación al primer plano. Nuestros roles en cuanto profesores deben cambiar radicalmente de manera que podamos concentrar nuestro tiempo y energías más en la evaluación formativa, en proporcionar feedback a nuestros alumnos que en explicarles la materia, ya que los alumnos tienen fácil acceso a muchas fuentes de información.

Efectivamente, el impacto en el aprendizaje de la forma de evaluar es tal que hace necesario que ésta se planifique con mucho detalle, atendiendo a todos los elementos que intervienen en el proceso: contexto, intenciones educativas (objetivos, contenidos, actividades, metodología...), proceso y resultados que se pretenden. En este sentido, como indica Cabrera (2003: 18), "la evaluación no se puede identificar con un único acto (pasar un cuestionario o pasar una prueba de rendimiento), sino con un proceso, o mejor dicho, con un conjunto de procesos no improvisados ni espontáneos". La evaluación requiere de una reflexión profunda en la que debemos plantearnos no solo qué va a ser evaluado y cómo, sino, sobre todo, para qué y cuándo, aspectos estos tradicionalmente desatendidos. Los cambios de la última década han Ilevado, afortunadamente, a preguntarnos qué finalidades tiene la evaluación y hasta qué punto la calificación -finalidad tradicional- ocupa una posición prioritaria e, incluso, si entre evaluación y calificación existe una relación de necesidad: ¿evaluar siempre lleva consigo calificar?; ¿se puede evaluar sin calificar?; ¿debe unirse la evaluación a la calificación? A estas alturas, está claro que la calificación es solo un aspecto más de la evaluación y, ni siquiera, un aspecto imprescindible. Sin embargo, sí nos parece del todo necesario que la evaluación cumpla, al menos, las siguientes finalidades:

- Ayudar a aprender, corregir errores a tiempo y evitar el fracaso.

- Condicionar un estilo de aprendizaje apropiado y eficaz.

- Favorecer un esfuerzo continuado durante todo el curso.

Por lo que respecta al momento en que debe llevarse a cabo la evaluación, ha de hacerse con frecuencia y estar perfectamente integrada en el proceso de aprendizaje. Esta es una característica fundamental si no queremos convertir la evaluación en un "añadido" y si pretendemos que realmente sirva a nuestros fines: ayudar a aprender, orientar la forma de hacerlo, etc. Estamos hablando, por tanto, de una evaluación formativa, integrada en la actividad académica como un elemento más del plan de trabajo, complementaria a los contenidos y actividades, contextualizada en el marco epistemológico de la asignatura y con un sentido y una finalidad (la evaluación también tiene que ser significativa). Siguiendo a Dunn y Mulvenon (2009), podemos definir la evaluación formativa como un conjunto de procedimientos de evaluación, tanto formales como informales, integrados en el proceso de enseñanza-aprendizaje y orientados a modificar y mejorar el aprendizaje y comprensión de los alumnos. Estos procedimientos aportan la información necesaria para ir ajustando el proceso a la consecución de los objetivos propuestos (Melmer, Burmaster y James, 2008). De esta manera, alumnos y profesores son conscientes en todo momento del progreso, las difi- 
cultades y los logros, y, en función de ello, pueden emprender las acciones convenientes sin tener que esperar al final del proceso (evaluación según la concepción tradicional) para obtener esa información, pues ¿de qué le sirve a un alumno saber que tiene un seis, por ejemplo, en un examen final?, e incluso, ¿de qué le sirve ver el examen y saber lo que no ha hecho bien? La evaluación formativa, sin embargo, da la oportunidad al alumno de aprender a partir de los errores, en tanto es consustancial a ella el análisis, la reflexión y la mejora del trabajo realizado. Y todo ello se logra gracias a la constante retroalimentación que el estudiante recibe durante el proceso.

¿Qué características debe tener la evaluación para que cumpla con tan ambiciosos objetivos? Puesto que la evaluación formativa se sustenta en el diálogo, la comprensión y la mejora (Santos Guerra, 1993), sus características vienen determinadas por estos tres aspectos. Así, siguiendo a Santos Guerra, cabe destacar su naturaleza integradora, en tanto que es el elemento que da cohesión y sirve de nexo de unión del resto de elementos del currículo (objetivos, contenidos, competencias, metodología...). Es, también, diversificada, ya que se vale de diferentes instrumentos para regular el proceso, evitando la repetición de tipos de pruebas y de medios. Es una actividad continua y sistemática que avanza de manera paralela al proceso de enseñanza y aprendizaje, analizándolo y reorientándolo cada vez que es necesario, planteando las soluciones durante el recorrido y no al final del camino, cuando ya no tienen sentido. Su principal finalidad es la formación, esto es, que los alumnos aprendan y mejoren de la forma adecuada tanto en el proceso como en los resultados. Caracteriza también la evaluación formativa su carácter criterial y técnico, pues parte de unos principios ajustados y acordes con la situación (no utiliza criterios universales y genéricos) y se vale de diferentes técnicas e instrumentos para aminorar los efectos de la subjetividad. En este sentido, es también independiente y práctica, ya que el profesor debe procurar la máxima neutralidad y no estar comprometido por otros factores ajenos a los especificados para el caso. Debe orientarse a la mejora del aprendizaje y no estar al servicio de exigencias o finalidades administrativas. Por último, la evaluación formativa debe ser democrática, participativa y personalizada, esto es, debe procurar un trato igualitario, no discriminador; ha de permitir la participación continua del alumno y atender a la diversidad del aula.

\section{UNA PROPUESTA DE EVALUACIÓN FORMATIVA INTEGRADA}

A partir del marco teórico descrito en el epígrafe anterior, decidimos llevar a las aulas universitarias algunos de los presupuestos básicos de la evaluación formativa integrada con objeto de valorar su factibilidad en nuestra asignatura, el impacto sobre el aprendizaje y, especialmente, su contribución a la resolución de algunas dificultades importantes con las que nos encontrábamos. Para ello, hemos diseñado una propuesta de trabajo que se ha desarrollado en la asignatura de Lengua Española de los Grados de Estudios Ingleses y de Lenguas Modernas y Traducción. Aunque son titulaciones distintas, la asignatura es común -y la misma- para todos. Se imparte durante el primer cuatrimestre, en horarios de mañana y tarde, y reúne un centenar de alumnos. Es de carácter obligatorio, de 8 créditos y con una escasa presencialidad. Los alumnos asisten a clase tres horas por semana, una de las cuales es en grupo grande (50 alumnos) y de naturaleza más o menos teórica, y las otras dos, en grupo pequeño (20 alumnos) y es práctica. 


\section{El contexto}

El interés por desarrollar una propuesta de innovación en evaluación surge como posible solución a una serie de problemas que nos venía planteando la asignatura:

- Falta de credibilidad entre los alumnos.

- Elevado número de estudiantes para un solo profesor.

- La amplitud del programa.

- La escasez de tiempo.

La asignatura de Lengua Española es una materia habitual en los planes de estudios desde el comienzo de la vida académica. Presenta una curiosa paradoja: cualquier individuo es consciente de su importancia, de su valor, de la necesidad de un aprendizaje constante, pero pocos encuentran sentido y utilidad a lo que en ella se enseña. Se trata de un problema que tiene su origen tanto en los contenidos (tradicionalmente los mismos, a pesar de los avances en Lingüística) como en la metodología seguida por maestros y profesores. Los alumnos llegan, por tanto, a la universidad sin tener un buen dominio sobre la lengua y sin creer que lo que van a estudiar les va a servir para mejorar. Esto contrasta con un hecho evidente: hay pocas asignaturas que sean tan competenciales como esta. Su sentido y finalidad en cualquier etapa es el desarrollo de la competencia comunicativa de los alumnos, entendida esta como la capacidad de una persona para comportarse lingüísticamente de manera eficaz y adecuada en una determinada comunidad de habla, lo que implica conocer y respetar un conjunto de reglas, que incluye tanto las de la gramática y los otros niveles de la descripción lingüística (léxico, fonética, semántica) como las reglas de uso de la lengua, relacionadas con el contexto social, histórico y cultural en el que tiene lugar la comunicación. Como señala Hymes (1996: 15).

La competencia comunicativa es el término más general para la capacidad comunicativa de una persona, capacidad que abarca tanto el conocimiento de la lengua como la habilidad para utilizarla. La adquisición de tal competencia está mediada por la experiencia social, las necesidades y motivaciones y la acción, que es a la vez una fuente renovada de motivaciones, necesidades y experiencias.

No es posible desarrollar destrezas y habilidades lingüísticas con la mera trasmisión de información. Parece evidente que para aprender a pintar, por ejemplo, es del todo insuficiente (e inútil) que le expliquen al alumno año tras año las características de los diferentes utensilios (caballete, pincel, óleos...) y las distintas técnicas. Y por más que esa información sea cada vez más exhaustiva y minuciosa, el mero conocimiento no le va a permitir dibujar correctamente un paisaje o hacer un buen retrato. De la misma manera, la descripción de la lengua, por más rigurosa y científica que sea, no le posibilitará convertirse en usuario competente. A los conocimientos se les debe dar un sentido de utilidad, y ese sentido se adquiere con la experiencia (tampoco sirve de nada que el profesor informe a sus alumnos de la utilidad de determinados contenidos). Por todo ello, las expectativas de los estudiantes con respecto a la asignatura son mínimas.

La asignatura se concibe como formación básica e instrumental para estos alumnos que serán, en el futuro, profesionales de la lengua (profesores, traductores, intérpretes, 
asesores lingüísticos...), por lo que está muy enfocada al desarrollo de una serie de competencias en comunicación lingüística. Creemos que queda fuera de toda duda la pertinencia e importancia de una asignatura que busca tanto formar hablantes altamente competentes como profesionales cuyo material de trabajo son, precisamente, las lenguas. A la hora de elaborar la propuesta hemos tenido muy claro que la asignatura debe apoyarse en el trabajo del alumno, en "aprender a hacer cosas", así como que los contenidos teóricos deben ser verdaderas ayudas para la consecución de las competencias marcadas. Para que esto fuera posible, ha sido necesario, entonces, llevar a cabo una selección drástica de contenidos, a pesar de lo cual el programa continuaba siendo de proporciones desmedidas. Piénsese que se recoge en un cuatrimestre escaso lo que en la licenciatura correspondiente se impartía en dos; la opción de tratar el programa completo pero con menor profundidad no nos parecía una solución válida, pues eso conduciría, en nuestro caso, a situarnos en etapas educativas pre-universitarias (bachillerato, incluso secundaria). La naturaleza de estos contenidos (los correspondientes a una asignatura general de Lengua Española), que se abordan desde secundaria, exige que, a medida que se avance, se profundice y se amplíen las dimensiones y perspectivas, pero casi no permite la adición de nuevos contenidos. Si examinamos los epígrafes generales del programa, veremos con rapidez que coinciden en gran parte con los de Lengua Española de Bachillerato, con el agravante de que en Bachillerato se trabajan durante dos años escolares y en el Grado, durante un cuatrimestre. Es fácil, entonces, entender que no es posible simplificar los contenidos, sino que la única opción está en reducirlos. A esto hay que añadir que, pese a que son temas que se tratan durante toda la vida escolar, el conocimiento y dominio que los alumnos tienen cuando llegan a las aulas universitarias es muy deficitario y poco útil.

En cuanto al tiempo del que se dispone, es del todo insuficiente teniendo en cuenta lo anteriormente señalado (amplitud del programa y pésimo nivel de los alumnos). Este problema se agrava si tenemos en cuenta que, de los ocho créditos de la asignatura, seis de ellos son no presenciales, lo que implica una enorme carga de trabajo para el alumno y, por ende, para el profesor, situación no siempre aceptada ni viable (según el caso). Pero es que, además, en la Facultad de Filosofía y Letras, los alumnos de primer curso de cualquier titulación suelen comenzar sus clases una o dos semanas después que el resto para poder acoger a los alumnos que hacen la PAU en septiembre. Esto nos deja con no más de 13 semanas reales de curso, esto es, 13 horas teóricas y 26 prácticas, para la misma asignatura que, ya bastante mermada, contaba anteriormente con 60 horas de teoría y 60 de prácticas.

Dadas las circunstancias en las que nos hallábamos, todo nos conducía a un plan de trabajo muy centrado en el alumno y sus necesidades, en el que éste se viera obligado a implicarse, a comprometerse y a adoptar un papel muy activo, y en el que el conocimiento, que debía ser muy significativo, se fuera realmente construyendo desde la práctica y la experiencia. Solo desde estas premisas podemos tratar de potenciar el trabajo de los alumnos fuera del aula (más cantidad que el de dentro) y lograr nuestros objetivos. Ello exige:

- Creación de escenarios de aprendizaje muy operativos y funcionales, lo que nos ha llevado al diseño de un conjunto amplio de actividades y tareas que nos aseguren la adquisición de los contenidos, destrezas y habilidades previstos. 
- Aplicación de distintas estrategias metodológicas que mantengan la motivación del alumno y permitan, al mismo tiempo, el logro de algunas competencias generales (capacidad de análisis y síntesis, de reflexión, de aprendizaje autónomo y cooperativo...).

- Seguimiento minucioso de todo el trabajo de los alumnos y retroalimentación constante. Este último aspecto es de enorme dificultad en situaciones como la nuestras, dado el número de alumnos -106 estudiantes en total-y requiere una dedicación del profesor a la asignatura casi exclusiva, ya que somos conscientes de la importancia del feedback dentro de nuestro planteamiento docente.

- Planificación de un sistema de evaluación, de carácter formativo e integrado y que concebimos, sobre todo, como estrategia para compensar algunas de las dificultades que hemos descritos y mejorar el aprendizaje. Lógicamente, el sistema de evaluación ideado también posibilita la calificación de la asignatura, requisito administrativo imprescindible.

\section{Descripción de la propuesta y metodología de trabajo}

En la propuesta que hemos desarrollado la evaluación se aborda desde una doble perspectiva: como procedimiento pluridimensional a partir del cual medir y calificar los resultados obtenidos, y como estrategia docente orientada a la mejora de la enseñanza y del aprendizaje.

- En su dimensión de procedimiento de calificación, la evaluación se realiza de la siguiente manera:

- Carpeta de trabajo: $40 \%$

- Trabajo de la asignatura: 40\%

- Asistencia, participación y compromiso: $20 \%$

La carpeta de trabajo está constituida por 17 actividades que se realizan a lo largo del curso, de diferente naturaleza pero con el mismo objetivo: hacer posible la adquisición y puesta en práctica de los contenidos fijados en el programa. Cada una de estas actividades debe entregarse en la fecha fijada para su corrección por parte del profesor. En ese momento, no se califican, solo se comentan y valoran. La mayor parte de ellas requiere trabajo en grupo y se realiza tanto en las horas de clases prácticas como fuera del aula. Hemos tratado en todo momento de que ese trabajo en grupo no resulte una división en partes, sino una práctica real de trabajo en colaboración.

Con respecto al trabajo de la asignatura, consiste en la realización de cuatro pequeñas tareas de investigación y de aplicación, muy dirigidas y supervisadas por el profesor durante las tutorías obligatorias en pequeños grupos. En este caso, es trabajo individual y se entrega solo a su finalización (en los Anexos puede verse una descripción de todas ellas).

- En cuanto a la dimensión formativa, la evaluación se desarrolla a partir de numerosos procedimientos, cada uno de ellos con unos objetivos concretos, unas dinámicas de trabajo determinadas y con distintas estrategias. Tienen en 
común su integración en el proceso como una actividad más, así como nuestra intención de hacer frente a alguno o varios de los problemas señalados.

Cada actividad y tarea de la carpeta de trabajo debe ser corregida por el profesor (no calificada) y devuelta a los alumnos para su revisión. Estos pueden entregarlas cuantas veces quieran hasta cerciorarse de que las han realizado correctamente. En este sentido, hay que señalar que el hecho de completar de forma adecuada la actividad implica haber adquirido determinadas habilidades, es decir, es evidencia de "haber aprendido a hacerlo".

Unidos muy directamente a estas actividades (a veces, integrados en ellas; otras, como parte final), se llevan a cabo numerosos ejercicios de autoevaluación y coevaluación orientados tanto a la reflexión sobre el proceso como a la sistematización o profundización en los contenidos. La evaluación se convierte, de esta manera, en una valiosa aliada con la que hacer frente a algunos de los inconvenientes descritos más arriba. Las dinámicas y técnicas para el desarrollo de estas actividades son muy variadas: test individuales de reflexión, debates en grupos pequeños a partir de un guión, corrección guiada de trabajos de otros compañeros, valoración y calificación argumentada de actividades (propias y ajenas)... En varias ocasiones, tras la realización de una actividad de la carpeta, se pide a los alumnos que analicen y comenten qué han hecho, cómo lo han hecho y para qué les puede servir. Eso los obliga a ir más allá del mero ejercicio y a encontrarle un sentido en relación con lo que saben y con las competencias que deben aprender. Indagar sobre la utilidad (esto es, sobre la aplicabilidad o el valor de transferencia) les permitirá entender y ver la asignatura -recuérdese: Lengua Española- desde un prisma hasta entonces desconocido. A veces, habrán de valorar y calificar los trabajos grupales presentados por los compañeros sin que puedan repetir la puntuación. Así, a partir de unos criterios de evaluación previamente consensuados, deben, en primer lugar, discutir en grupo la calidad de los trabajos presentados; después, valorar cualitativamente los diferentes apartados y, por último, repartir las calificaciones entre los grupos (A, B y $C$, si eran tres grupos; A, B, C y D si eran cuatro, etc.). La decisión debe estar mínimamente motivada. Este tipo de actividad ayuda de forma notable a afianzar los contenidos presentados, pues obliga a volver sobre ellos, a compararlos y a entenderlos. En otros casos, se llevarán a cabo ejercicios individuales que pretenden generar una reflexión sobre las dificultades encontradas, la razón de esas dificultades, posibles formas de hacer frente... y al mismo tiempo, los logros, los aspectos que se dominan, etc. Muchos alumnos nos han comentado que la propia reflexión y descripción de tales aspectos han sido la clave para entenderlos o ha sido un aliciente para profundizar un poco más y plantearse nuevas metas. La puesta en común de estas reflexiones se convierte, también en elemento especialmente valioso para trabajar los contenidos del programa.

Con el trabajo de la asignatura se sigue una dinámica similar, aunque en este caso, se fomenta especialmente la reflexión metacognitiva y la autoevaluación. De nuevo hemos intentado que las secuencias didácticas que forman las ta- 
reas incluyan ejercicios de evaluación integrados en la secuencia y contextualizados a partir de los contenidos que se trabajan.

La implicación de los alumnos en el proceso se valora tanto por la realización de todas las actividades propuestas, independientemente de su naturaleza y finalidad (de adquisición de contenidos, de desarrollo de competencias, de evaluación...) como por su participación y compromiso. El análisis de estos últimos aspectos se sustenta en la observación por parte del profesor y en algunas tareas de autoevaluación y coevaluación. Así, por ejemplo, tras la realización de alguna actividad grupal se pide a los alumnos que comenten el funcionamiento del equipo, la aportación de cada miembro, las ventajas e inconvenientes de la cooperación. En otros casos, tienen que defender ante el grupo la labor realizada y la importancia de su trabajo para el cumplimiento de la tarea (dentro del marco de los contenidos y objetivos del programa). Al final, cada uno tiene que votar por el miembro del grupo que, en su opinión, ha destacado en la tarea. Como en otras ocasiones, la decisión debe argumentarse en tres o cuatro líneas.

\section{ReSULTADOS DE LA EXPERIENCIA}

La implementación del sistema de evaluación descrito fue valorado muy positivamente por los alumnos. No obstante, nuestro objetivo no era aumentar el grado de satisfacción sino resolver serias dificultades que estaban condenando la asignatura al exilio de las "marías". A pesar de nuestros esfuerzos de años anteriores por una enseñanza de calidad, motivadora y rigurosa, éramos conscientes de su ineficacia, al menos, así lo percibíamos desde nuestros planteamientos docentes.

Creemos haber alcanzado los objetivos de partida; podemos señalar como logros de esta experiencia de innovación los siguientes:

- Hemos conseguido compensar la escasez de contenidos así como la superficialidad con que nos veíamos obligados a trabajarlos. Las tareas de evaluación generaron oportunidades para abordar aspectos nuevos en cada tema y, sobre todo, profundizar más en todos ellos.

- Nos hemos asegurado un aprendizaje duradero y de calidad, con sentido y fácilmente transferible. La transferencia del conocimiento solo es posible cuando se toma conciencia de su potencialidad; por su parte, la toma de conciencia solo se da desde la propia experiencia y reflexión. La evaluación formativa ha favorecido notablemente los procesos que conducen al desarrollo de un pensamiento crítico, a la reflexión y análisis como forma de llegar al conocimiento y a la aplicación fundamentada y razonada del saber.

- Hemos logrado aumentar considerablemente el esfuerzo y trabajo continuado del alumno fuera del aula, imprescindible para cumplir tanto con los objetivos como con la carga lectiva asignada. Puesto que la calificación se apoyaría exclusivamente en los resultados del conjunto de actividades y tareas, los alumnos han querido asegurarse el éxito de la asignatura elaborando todo el trabajo y mejorándolo en todo lo posible. Gracias a ello, el escaso tiempo de 
actividad académica presencial se ha visto bastante ampliado con el trabajo realizado fuera del aula.

- Hemos conseguido que los alumnos entiendan la asignatura como elemento de formación indispensable en sus estudios, que valoren sus aportaciones para el desempeño profesional futuro. $Y$, sobre todo, que entiendan la relación existente entre determinados conocimientos teóricos y el desarrollo de la competencia comunicativa.

- Los alumnos han adquirido conciencia del proceso de aprendizaje, de su naturaleza, de los elementos que participan, etc.; han asumido un papel activo y responsable; han mejorado sus aptitudes para trabajar en equipo, así como los resultados obtenidos del trabajo en cooperación.

\section{ASPECTOS DE MEJORA}

Aunque estamos convencidos de que esta misma experiencia docente podría implementarse en muchas asignaturas de otras áreas de conocimiento, sería conveniente comprobar su eficacia más allá de nuestra titulación.

Otro aspecto en el que habría que seguir trabajando es el del papel que cumplen la autoevaluación y coevaluación. En nuestra propuesta, los resultados de este tipo de actividades solo se han tenido en cuenta para valorar la implicación y el compromiso, y se ha hecho de una manera bastante impresionista y subjetiva, puramente cualitativa. Sí han resultado de enorme utilidad, como ya se ha comentado, para incidir en los contenidos, mejorar las formas de aprender, etc. Pero nos parece que pueden cumplir más funciones, que son susceptibles de un mayor rendimiento. Tal vez si adquirieran mayor protagonismo y se orientaran más a la calificación, podría disminuir en alguna medida el trabajo del profesor, que reduciría la cantidad de actividades finales para calificar.

Sin duda, el principal inconveniente de la propuesta llevada a cabo ha sido el volumen de trabajo que ha supuesto para el profesor, originado, sobre todo, por las correcciones (con comentarios incluidos) de las numerosísimas actividades. Ha sido necesario dedicarse casi exclusivamente a esta asignatura, desatendiendo otras tareas y funciones propias del profesor universitario. En este sentido, la propuesta requiere de las modificaciones necesarias para que sea compatible con el desarrollo de otras actividades. Una buena solución es sustituir la carpeta de trabajo como instrumento de calificación por un portafolio en el que se recojan solo algunas de las actividades realizadas por el alumno. A diferencia de la primera, el portafolio se conforma a partir de una selección de trabajos realizada por el alumno o por el alumno y el profesor de acuerdo con unos criterios de calidad, y tiene la finalidad de documentar las capacidades adquiridas y el progreso a lo largo del curso. Además de los trabajos, el portafolio debe incluir las razones de la selección, los criterios para juzgar el mérito, las reflexiones del alumno, etc. La elección debe hacerse siempre con un propósito definido, lo que aporta un marco concreto sobre el que valorar, juzgar y seleccionar. Las finalidades pueden ser muy diferentes: los trabajos que mejor ilustren la evolución del alumno en una determinada competencia o capacidad; los que resulten de mayor calidad; los que le han supuesto un mejor aprendizaje. Esto obliga al alumno a reflexionar sobre el proceso que ha 
seguido. Él es consciente de que será calificado por los trabajos que escoja, por lo que es el más interesado en ajustarse en todo lo posible a los criterios de evaluación establecidos. Estos criterios, lógicamente, deben responder a los objetivos plasmados en el programa, por lo que la selección implica también conocer bien esos objetivos y sus formas de desarrollo. Todo ello hace que los portafolios se conviertan en instrumentos de autoevaluación de alta calidad, ya que se hace con una finalidad ulterior, lo que lleva al alumno a ser consciente de sus logros, sus carencias, sus dificultades... siempre en relación a unos objetivos y a unas competencias que actúan como marco de evaluación. Como señalan Danielson y Abrutyn (1995), el procedimiento de seleccionar trabajos con una finalidad determinada combina los procesos de enseñanza y evaluación, y esa es la característica que hace del portafolio un instrumento educativo poderoso. El portafolio no solo disminuirá la cantidad de correcciones finales, sino que su propia elaboración constituiría una forma más, de enorme valor, de hacer de la evaluación verdaderas oportunidades para aprender.

\section{Conclusiones}

La evaluación no puede entenderse como un simple procedimiento para calificar a los alumnos, pues su impacto sobre el aprendizaje es determinante. Por otra parte, puede ayudar notablemente a la mejora del proceso de enseñanzaaprendizaje al proporcionar información de manera continua tanto a alumnos como a profesores. Así lo demuestran numerosísimos estudios realizados en todos los niveles educativos, en las diferentes áreas de conocimiento y en las más diversas situaciones educativas. Pero, además, si se integra en el trabajo que ha de realizar el alumno y se la dota de contexto, contenido y finalidad (más allá de la propia valoración del proceso) puede convertirse en una herramienta de gran utilidad para el desarrollo de competencias y contenidos específicos de la materia. Todo ello lo hemos podido comprobar en la experiencia de innovación llevada a cabo en la asignatura de Lengua Española de los Grados de Estudios Ingleses y de Lenguas Modernas y Traducción de la Universidad de Alcalá. El sistema de evaluación (de carácter formativo e integrado) nos ha permitido profundizar en los contenidos, favorecer un aprendizaje significativo, potenciar la reflexión, la investigación y el pensamiento crítico; al mismo tiempo, ha hecho posible que hiciéramos frente a algunos problemas con los que nos encontrábamos, como la falta de credibilidad de la asignatura, la escasez de tiempo, la poca presencialidad, la enorme carga de trabajo autónomo o la desmotivación.

\section{REFERENCIAS BIBLIOGRÁFICAS}

BROWN, S. (2006). Assessment is the most important thing we do for HE students. London: The Open University.

CABRERA, A. F. (2003). Evaluación de la formación. Madrid: Síntesis.

DANIELSON, Ch. y ABRUTYN, L. (1997). An introduction to using portfolios in the classroom. Alexandria: Association for Supervision and Curriculum Development. 
DUNN, K. E. y MULVENON, S. W. (2009). A Critical Review of Research on Formative Assessments: The Limited Scientific Evidence of the Impact of Formative Assessments in Education. Practical Assessment Research \& Evaluation, 14 (7). Disponible en: http://pareonline.net/getvn.asp? $v=14 \& n=7$ (consultado el 12 de junio de 2010).

ELTON, L. R. B. y LAURILLARD, D. M. (1979). Trends in Research on Student Learning. Studies in Higher Education, 4, 87-102. Disponible en: http://stadium. open.ac.uk/perspectives/assessment (consultado el 7 de junio de 2010).

HYMES, D. H. (1971). On communicative competence. Philadelphia: University of Pennsylvania Press. Versión en español: Hymes, D. (1996). Acerca de la competencia comunicativa. Forma y función (9), 13-37.

KEMBER, D. (2000). Misconceptions about learning approaches, motivation and study practices of Asian students. Higher Education, 40, 99-121.

MELMER, R.; BURMASTER, E. y JAMES, T. K. (2008). Attributes of effective formative assessment. Washington, DC: Council of Chief State School Officers. Disponible en: http://www.ccsso.org/publications/details.cfm?PublicationID=362 (consultado el 12 de junio de 2010).

SANTOS GUERRA, M. A. (1993). La evaluación, un proceso de diálogo, comprensión y mejora. Archidona: Aljibe.

\section{ANEXOS}

\section{Anexo I. Actividades de evaluación}

1. Tras la realización de una actividad grupal de cierta complejidad (actividad 1: tipología discursiva), deben asignar un punto al compañero que, por la razón que sea, crean que ha realizado el mejor trabajo. El profesor les sugiere motivos: mejores aportaciones, organización del trabajo, gestión del grupo, conocimientos... Han de hacerlo por escrito y razonar su decisión.

2. Reflexión grupal guiada. Tras la realización de una actividad basada en la estrategia del alumno experto, el grupo reflexiona y contesta por escrito a las siguientes cuestiones:

- ¿En qué ha consistido el trabajo?, ¿qué han tenido que hacer?

- ¿Para qué lo han hecho?

- ¿Qué han aprendido o trabajado, además de unos contenidos concretos de la materia?

- ¿Cómo/dónde pueden utilizar lo que han aprendido?

- Relacionar los aspectos anteriores con objetivos, competencias y contenidos de la guía docente.

3. Valoración individual de las presentaciones de sus compañeros y reflexión grupal. Para la valoración individual, cuentan con una tabla que recoge los criterios de valoración, previamente consensuados (claridad expositiva, pertinencia e interés de la información seleccionada, coordinación del grupo, dominio del tema -que se comprueba a través de las preguntas-, utilización de los recursos...). Al final deben reunirse por grupos, comentar sus valoraciones y, de común acuerdo, deben repartir las puntuaciones $A, B$, C y $\mathrm{D}$ entre ellos, sin que pueda repetirse ninguna de ellas (a cada grupo deben asignarle una puntuación distinta). Deben razonar su decisión. 
4. Corrección y valoración entre grupos. Tras la realización de una actividad en grupo, cada uno de ellos debe pasar a otro grupo su trabajo para su corrección y valoración. Esta corrección se hace individualmente, pero se comparte. Esto es, un alumno lee el trabajo, hace los comentarios oportunos y pasa el trabajo y la hoja de evaluación a otro compañero de su grupo; éste, lo lee, corrige y valora a partir de lo que está escrito; pasa al tercer miembro del grupo, que repite las acciones anteriores. Los alumnos cuentan con una guía de evaluación:

- Lo mejor del trabajo es... porque............

- Sería conveniente mejorar. porque

- En comparación con nuestro trabajo...........

- ¿Lo nominaría para ser elegido el mejor trabajo del cuatrimestre? ¿Por qué?

5. Tras una práctica grupal de análisis y clasificación de errores lingüísticos, de manera individual deben revisar las tareas corregidas por el profesor y analizar sus propios errores, reflexionando sobre la calidad de sus escritos, otorgándose una puntuación y proponiendo acciones para la subsanación y mejora.

6. En grupo, a partir de una selección de actividades y tareas realizadas en el curso, relacionar cada una con los objetivos y contenidos del curso. De manera individual, contestar un test sobre el mayor o menor cumplimento de las competencias generales y específicas previstas en el curso. Se pide también argumentar y explicar algunos de los casos.

\section{Anexo II. Carpeta de trabajo: actividades para la adquisición o práctica de los contenidos de la asignatura}

\begin{tabular}{|c|c|}
\hline $\begin{array}{l}\text { A. Tipología discursiva } \\
\text { (narración, } \\
\text { descripción, } \\
\text { exposición y } \\
\text { argumentación) }\end{array}$ & $\begin{array}{l}\text { 1. Tipos de discurso y comentario lingüístico de textos. Se elabo- } \\
\text { ra un dossier informativo por cada tipo de discurso. Se frag- } \\
\text { mentan en partes (tantas como alumnos haya en el grupo; } \\
\text { nunca más de 20) y se reparten. Cada uno lee y resume su } \\
\text { parte. Los alumnos se agrupan en función del tipo de discurso, } \\
\text { explican la parte que les ha tocado y entre todos, prepararan } \\
\text { un nuevo documento, resumido, con los aspectos fundamen- } \\
\text { tales. Se forman nuevos grupos (en cada uno tiene que haber } \\
\text { un representante de cada tipo de discurso) y cada alumno, que } \\
\text { ya es "experto" en un tipo de discurso, explica a los demás su } \\
\text { modalidad. Tras las exposiciones, se les entrega seis textos para } \\
\text { que los comenten desde el punto de vista lingüístico. }\end{array}$ \\
\hline \multirow[b]{2}{*}{ B. Fonética y fonología } & $\begin{array}{l}\text { 2. Definiciones de conceptos básicos de fonética y fonología. Se } \\
\text { entrega a los alumnos textos sencillos con explicaciones } \\
\text { (deberán evitarse las definiciones explícitas) sobre conceptos } \\
\text { fundamentales para que, en parejas, traten de definirlos de la } \\
\text { manera más técnica y precisa posible. }\end{array}$ \\
\hline & $\begin{array}{l}\text { 3. Clasificación en cuadro fonético de los alófonos del español. } \\
\text { Se entrega a los alumnos información con la descripción de los } \\
\text { fonemas y alófonos del español. A partir de esa información, } \\
\text { deben cumplimentar un cuadro de clasificación. Después, con } \\
\text { el cuadro de clasificación (solo con el cuadro), deben caracte- } \\
\text { rizar cada fonema (ej. /b/ consonántico, oral, bilabial, oclusi- } \\
\text { vo, sonoro). }\end{array}$ \\
\hline
\end{tabular}




\begin{tabular}{|c|c|}
\hline B. Fonética y fonología & $\begin{array}{l}\text { 4. Comparación del sistema fonético del inglés y del español. } \\
\text { Se proporciona a los alumnos información básica sobre el } \\
\text { sistema fonológico del inglés. En grupo, cada uno de ellos se } \\
\text { ocupa de un tipo (p. ej, las fricativas). Comparan ambos sis- } \\
\text { temas y realizan una exposición ante el resto de los compa- } \\
\text { ñeros. Deben proporcionales la información conveniente, } \\
\text { pues, al final, cada uno tendrá que tener la comparación de } \\
\text { los dos sistemas completos. }\end{array}$ \\
\hline \multirow{3}{*}{$\begin{array}{l}\text { C. Lexicología y } \\
\text { semántica }\end{array}$} & $\begin{array}{l}\text { 5. Impropiedades léxicas. Se proporciona a cada alumno una } \\
\text { lista de frases (60) sacadas de medios de comunicación, publi- } \\
\text { cidad, etc. en el que se hace un uso incorrecto de un término } \\
\text { (p. ej. Es una persona asequible, por Es una persona accesi- } \\
\text { ble). De forma individual o en parejas deben subsanar el error } \\
\text { y explicar la confusión. La consulta a diccionarios (DRAE, } \\
\text { Diccionario de panhispánico de dudas...) será obligatoria. }\end{array}$ \\
\hline & $\begin{array}{l}\text { 6. Pobreza léxica. Se entrega a los alumnos cinco tablas con ver- } \\
\text { bos "comodines" (hacer, tener, haber, dar, poner), cada una de } \\
\text { ellas con, aproximadamente, } 15 \text { expresiones imprecisas (p. ej. } \\
\text { poner la firma, hacer estragos, tener un cargo...); deben buscar } \\
\text { la forma conveniente. Se puede plantear a modo de juego entre } \\
\text { ellos: cada uno tiene las soluciones de una tabla. Se trata de ver } \\
\text { quién encuentra más soluciones. Después, por grupos, deben } \\
\text { construir un relato de ficción en el que se incluyan todas las } \\
\text { expresiones trabajadas en las tablas. Para ello, deben ponerse } \\
\text { de acuerdo sobre el tema y el argumento y después, a modo de } \\
\text { "libro viajero" cada uno debe ir completando un capítulo. }\end{array}$ \\
\hline & $\begin{array}{l}\text { 7. Diccionarios. El profesor presenta los tipos de diccionarios } \\
\text { más habituales (de dudas, terminológicos, etimológicos, de } \\
\text { americanismos, académico, escolar...). Cada grupo debe } \\
\text { elegir un tipo, preparar una presentación y elaborar una pro- } \\
\text { puesta de actividades para trabajar con él. Si se dispone de } \\
\text { tiempo, lo ideal es realizar en clases esas actividades. }\end{array}$ \\
\hline \multirow[b]{2}{*}{ D. Morfología } & 8. Formación de palabras: derivados. \\
\hline & $\begin{array}{l}\text { 9. Formación de palabras: compuestos. Se realiza una serie de } \\
\text { actividades de preparación para conocer los tipos de compues- } \\
\text { tos. Después, en grupos, y a partir de todos los ejemplos vistos } \\
\text { en los ejercicios y otros que puedan proponer, deben elaborar, } \\
\text { sin consultar fuentes, una explicación clara y concisa sobre la } \\
\text { formación de femeninos y plurales en las palabras compuestas. }\end{array}$ \\
\hline E. Gramática & $\begin{array}{l}\text { 10. Concepciones sobre la gramática y su valor. Se proporciona } \\
\text { a los alumnos seis textos con opiniones y puntos de vista de } \\
\text { distintos autores sobre el valor de la gramática, los tipos de } \\
\text { gramática, etc. Se leen individualmente y entre todos, se van } \\
\text { analizando los distintos temas que se plantean en cada caso, } \\
\text { su relación con otros aspectos estudiados... }\end{array}$ \\
\hline
\end{tabular}




\begin{tabular}{|c|c|}
\hline \multirow{5}{*}{ E. Gramática } & $\begin{array}{l}\text { 11. Diferencias entre la gramática de la L1 y la de la L2. A par- } \\
\text { tir de un cuestionario, se guía la reflexión de los alumnos } \\
\text { en torno a las diferencias entre ellas, sobre su adquisición } \\
\text { y aprendizaje, sobre el desarrollo de la competencia lin- } \\
\text { güística, etc. }\end{array}$ \\
\hline & $\begin{array}{l}\text { 12. Rasgos característicos de las diferentes categorías gramati- } \\
\text { cales. Se proporciona a los alumnos una lista con numero- } \\
\text { sos rasgos de categorías gramaticales (p. ej. Señalan la } \\
\text { cercanía o lejanía de la realidad designada por el nombre } \\
\text { al que acompañan con respecto al hablante). Deben rela- } \\
\text { cionar cada rasgo con la categoría correspondiente. Pue- } \\
\text { den consultar las gramáticas recomendadas por el profesor. }\end{array}$ \\
\hline & $\begin{array}{l}\text { 13. Análisis de textos a partir de las categorías presentes en él. } \\
\text { Se proporciona a los alumnos cinco o seis textos para que } \\
\text { analicen las categorías predominantes y extraigan conclu- } \\
\text { siones respecto a la naturaleza del texto. }\end{array}$ \\
\hline & $\begin{array}{l}\text { 14. Conceptos de oración y de palabra. Se presenta un docu- } \\
\text { mento con las definiciones de oración y palabra más } \\
\text { importantes o que han tenido mayor trascendencia. El pro- } \\
\text { fesor explica, sin referirse a ninguna definición en concre- } \\
\text { to, objeciones o inconvenientes y los estudiantes van } \\
\text { tomando nota. Después, en grupo, deben analizar qué } \\
\text { objeciones pueden ponerse a cada definición y deben indi- } \\
\text { car cuál de las definiciones les parece más acertada y por } \\
\text { qué. Se hará puesta en común. }\end{array}$ \\
\hline & $\begin{array}{l}\text { 15. Lectura y comentario crítico de dos artículos sobre el géne- } \\
\text { ro y el número en español. Se remite a los alumnos a la } \\
\text { página web de la RAE para que lean con detenimiento los } \\
\text { artículos que se recogen sobre el género y el número en } \\
\text { español. Deben hacer una reseña. }\end{array}$ \\
\hline \multirow{2}{*}{ F. Lengua y norma } & $\begin{array}{l}\text { 16. Actividad de iniciación a la investigación que consiste en la } \\
\text { identificación de errores lingüísticos en escritos y en la elabo- } \\
\text { ración de una tipología. El profesor proporciona a los alumnos } \\
\text { textos reales de diferente tipología escritos por compañeros de } \\
\text { la universidad o por estudiantes de bachillerato. En parejas o } \\
\text { en grupos, deben identificar el error, explicarlo y corregirlo. } \\
\text { Después, han de elaborar una tipología de errores. }\end{array}$ \\
\hline & $\begin{array}{l}\text { 17. Identificación de los propios errores y elaboración de } \\
\text { fichas explicativas. A partir de todos las actividades entre- } \\
\text { gadas al profesor y corregidas por él, deben extraer una } \\
\text { lista de los errores más recurrentes y preparar para cada } \\
\text { uno una ficha que les pueda ayudar a entender el proble- } \\
\text { ma y, sobre todo, a solucionarlo. }\end{array}$ \\
\hline
\end{tabular}




\section{Anexo III. Trabajo del curso: actividades para la adquisición de contenidos que no se abordan durante las sesiones de clase}

\section{Lenguaje periodístico}

- Búsqueda de las características más importantes del lenguaje periodístico.

- Ejemplificación de esas características a partir de una o varias noticias.

- Análisis de la noticia.

- Redacción de una noticia.

- La manipulación de la información. Recursos para mostrar el punto de vista en la noticia.

- Redacción de una noticia desde dos puntos de vista distintos.

\section{Lenguaje científico-técnico}

- A partir de las características que proporciona el profesor, analizar y ejemplificar con varios textos científicos.

- Transformar textos divulgativos en otros de carácter más técnico o científico.

- Analizar los recursos para la formación de palabras y ejemplificar con términos de, al menos, cinco ámbitos distintos.

- Estrategias para la lectura y comprensión de textos científicos.

\section{Lenguaje de divulgación}

- La reformulación: análisis de los elementos reformuladores en varios textos proporcionados por el profesor.

- Analizar los tipos de textos y sus destinatarios.

- Analizar los recursos (lingüísticos, textuales, tipográficos) fundamentales de los textos didácticos.

- Transformar un texto científico en otro de divulgación de carácter didáctico.

\section{Lenguaje académico}

- Lectura de la información sobre los lenguajes académicos preparada por el profesor.

- Tras escuchar la presentación que el profesor hace sobre un pequeño proyecto de investigación que pretende llevar a cabo ("Problemas expresivos de los adolescentes"), los alumnos deben tomar nota y redactar ese proyecto siguiendo el siguiente esquema: introducción, objetivos, marco teórico, metodología de la investigación y bibliografía básica. 